\title{
Musculoskeletal responses of spinal cord injured individuals to functional neuromuscular stimulation-induced knee extension exercise training
}

Mary M. Rodgers, PhD, PT; Roger M. Glaser, PhD; Stephen F. Figoni, PhD, RKT; Steven P. Hooker, PhD; Bertram N. Ezenwa, PhD; Steven R. Collins, MS; Thomas Mathews, MD; Agaram G. Suryaprasad, MD; Satyendra C. Gupta, MD Department of Veterans Affairs Medical Center, Dayton, $O H$ 45428; Institute for Rehabilitation Research and Medicine, Wright State University School of Medicine, Dayton, OH 45435; Rehabilitation Institute of Ohio, Miami Valley Hospital, Dayton, OH 45409

\begin{abstract}
This study was conducted to evaluate a newly designed functional neuromuscular stimulation (FNS)-induced knee extension (KE) exercise system that incorporates the most desired features of previously described systems by determining the musculoskeletal responses of spinal cord injured (SCI) individuals to training. A specially designed chair and electrical stimulator were fabricated for FNS-induced KE resistance exercise. Surface electrodes were placed over motor points of the quadriceps muscles, and $\mathrm{KE}$ was alternated between legs at an average rate of $6 \mathrm{KE} / \mathrm{min} / \mathrm{leg}$. $\mathrm{KE}$ testing protocols were developed for pre- and post-training evaluations of performance, and $12 \mathrm{SCI}$ subjects exercise-trained up to three times per week for 36 sessions using a progressive resistance load at ankle level. Pre- and post-training evaluation data were statistically compared using a 0.05 level for significance. Quadriceps muscle performance (strength $\times$ repetitions) improved for both legs in all subjects as indicated by significant increases in load resistance and repetitions over the 36-session training period (right leg $\bar{x}=1156.0$ versus $1624.8 \mathrm{~kg} \cdot$ reps, left leg $\bar{x}=1127.3$ versus $1721.1 \mathrm{~kg} \cdot \mathrm{reps})$. In addition, knee range of motion significantly increased (right leg $\bar{x}=134$ versus 146 degrees, left leg $\bar{x}=133$ versus 144 degrees). Thigh skinfold, thigh girth, body
\end{abstract}

Address all correspondence and requests for reprints to: Mary M. Rodgers, PhD, PT, Department of Rehabilitation Medicine and Restorative Care, Wright State University Research Center, 3171 Research Boulevard, Kettering, OH 45420. This work was supported by the Rehabilitation Research and Development Service of the U.S. Department of Veterans Affairs. weight and bone density were not significantly changed. The lack of decrease in bone density in some subjects suggests that the training may retard the rate of bone loss which typically occurs with SCI. No injuries or problems were encountered during testing and training. Therefore, this newly-designed FNS$\mathrm{KE}$ exercise appears to be safe, easy to use, and effective for all subjects who participated in the study. The protocol developed for this form of FNS training of the quadriceps may be used to increase the integrity of muscles paralyzed due to $\mathrm{SCI}$. In addition, the enhanced muscle performance reserve may better enable the SCI individual to accomplish more complex FNS tasks with less fatigue.

Key words: functional neuromuscular stimulation (FNS), knee extension exercise, muscle performance, paralyzed quadriceps muscle, spinal cord injury (SCI), therapeutic exercise.

\section{INTRODUCTION}

Although traditional therapeutic exercise for spinal cord injured (SCI) individuals has primarily centered upon upper extremity resistance training to improve muscle strength and endurance $(2,15)$, functional neuromuscular stimulation (FNS) of paralyzed lower extremity muscles has been increasingly used in the rehabilitation process $(9,10,13,18,19,20,22)$. Improved strength and endurance of 
Table 1.

Description of subjects.

\begin{tabular}{lccccc}
\hline Sex & $\begin{array}{c}\text { Age } \\
(\mathrm{yr})\end{array}$ & $\begin{array}{c}\text { TSI } \\
(\mathrm{yr})\end{array}$ & $\begin{array}{c}\text { Weight } \\
(\mathrm{cm})\end{array}$ & $\begin{array}{c}\text { Weight } \\
(\mathrm{kg})\end{array}$ & \multicolumn{1}{c}{ Lesion } \\
\hline female & 19 & 0.8 & 160 & 48 & $\mathrm{C}_{5}$ incomplete \\
male & 22 & 0.7 & 185 & 72 & $\mathrm{~T}_{10}$ incomplete \\
male & 28 & 5.0 & 174 & 62 & $\mathrm{C}_{5}$ complete \\
male & 33 & 1.7 & 172 & 61 & $\mathrm{C}_{6}$ incomplete \\
male & 34 & 15.4 & 183 & 70 & $\mathrm{C}_{6}$ complete \\
male & 37 & 4.7 & 177 & 75 & $\mathrm{C}_{4}$ incomplete \\
female & 38 & 4.1 & 170 & 68 & $\mathrm{~T}_{1}$ complete \\
female & 41 & 1.6 & 173 & 77 & $\mathrm{C}_{5}$ incomplete \\
male & 41 & 15.3 & 170 & 80 & $\mathrm{C}_{7}$ incomplete \\
male & 48 & 2.4 & 170 & 54 & $\mathrm{~T}_{6}$ complete \\
male & 56 & 7.7 & 188 & 84 & $\mathrm{C}_{7}$ complete \\
male & 63 & 17.0 & 175 & 67 & $\mathrm{~T}_{5}$ incomplete \\
\hline $\bar{X}$ & 38.3 & 6.4 & 174.7 & 68.1 & \\
\pm SD & \pm 12.9 & \pm 6.1 & \pm 7.7 & \pm 10.7 & \\
\hline
\end{tabular}

paralyzed quadriceps muscles have been reported by various research groups using FNS knee extension (KE) exercise $(5,7,11,21)$. However, the instrumentation used for these studies was quite diverse with respect to the FNS control technique, the method of resistance application, and the timing/patterns of the contractions. Each of the FNS-KE systems described in the literature had advantages and disadvantages in their design strategies and each may produce somewhat different training results. Therefore, if FNS-KE exercies is to be made widely available for clinical and home use, the best feature of each of the previous designs need to be incorporated in the instrumentation. In addition to utilizing different types of instrumentation, previous studies implemented different training protocols, methods of evaluation, and durations of training ( 4 to 30 weeks). The variety of methods used in these studies makes assessment of the effectiveness of FNS-KE training difficult to interpret. Therefore, there is a need to develop more standardized protocols and evaluation procedures to better document FNS-KE training responses, as well as to develop an optimal equipment design so that the FNS-KE exercise can be performed in a safe, effective, convenient, and inexpensive manner.

In the present work, the positive aspects of previous instrumentation and protocols have been incorporated to produce a composite FNS-KE system which alleviates most identified inadequacies. These features have been discussed in Ezenwa, et al. (3). Acute physiologic responses using this system have also been reported (6). The purpose of the current study was to further evaluate this FNS-KE system and a training protocol by determining the musculoskeletal effects of this exercise mode for SCI individuals. (See Appendix for summary of abbreviations.)

\section{METHODS}

\section{Subjects}

SCI individuals were recruited from the Dayton, Ohio area for this study. All participants signed statements of informed consent approved by the Wright State University Institutional Review Board prior to their involvement in the research. Extensive medical screening was performed for each subject in order to identify any contraindications to participation in FNS exercise. These medical evaluations included blood analysis, urinalysis, psychological and neurological testing, and X-rays of lower extremities and chest. In addition, a detailed medical history and physical examination were conducted by a cardiologist for each participant. Only those with medical clearance were allowed to participate in the study. Contraindications to participation in FNS exercise included lower motoneuron involvement, previous lower extremity fracture, medical and/ or psychological instability, and intolerance to electrical stimulation.

Twelve SCI individuals qualified as subjects for this study. Included in this group were eight quadriplegics (three neurologically complete), and four paraplegics (two neurologically complete). Subject characteristics are given in Table 1. All subjects were asked to maintain their pretraining general activity levels while participating in the study. One subject (34-year-old male, 16 years post $\mathrm{C}_{6}$ complete $\mathrm{SCD}$ had previously been involved with FNS research projects (FES leg cycle ergometry) prior to this study. For safety reasons, the medication schedules of the subjects were not altered during this study. Seven of the 12 subjects took antispasticity medications (Valium, Lioresal, Ditropan, or Dantrium) during the training period. Other medications were not related to musculoskeletal function.

\section{FNS-KE instrumentation}

The chair and electrical stimulator system used to induce FNS-KE exercise includes unique instrumentation features which have been previously described in detail $(3,4)$. In summary, this system enabled asynchronous leftright $\mathrm{KE}$ exercise through approximately 70 degrees at a 
rate of approximately $6 \mathrm{KE} / \mathrm{min} / \mathrm{leg}$. External resistance was applied via load weights placed upon the lever arms at ankle level. The stimulation current was automatically ramped up and down to allow safe and smooth concentric and eccentric contractions. A knee angle-feedback system, incorporating potentiometer and microswitch sensors in the lever arms, ensured appropriate range of KE motion. In addition, threshold current and stimulation output current ramp parameters were continuously adapted (with each contraction) to maintain appropriate range of $\mathrm{KE}$ motion as fatigue occurred. Knee joint hyperextension, which can occur with spasticity, can be detected with this system and would initiate a reduction of stimulation current. The chair back was fixed to maintain a hip angle of approximately 110 degrees. This hip angle reduced stretching of the hamstring muscles, thereby minimizing resistance to knee extension from spastic or tight hamstrings. Strap stabilization at the hips, knees, and ankles was used to maintain joint alignment during exercise.

\section{Musculoskeletal evaluations}

Pre- and post-training musculoskeletal evaluations included passive knee range of motion (ROM), thigh skinfold thickness, thigh girth, body weight, and maximum $\mathrm{KE}$ load resistance capability. Trabecular bone density was measured at the distal end of the tibia using a specialized quantitative computed tomography technique which has been previously described (12). Passive knee range of motion was measured with the subject in the prone position using a standard goniometer. Thigh skinfold thicknesses and girths were measured with the subject in the supine position. Thigh skinfold was measured using Lange skinfold calipers at the anterior mid-thigh level. Thigh girths were measured at proximal, middle, and distal thirds of the thigh length using a flexible tape measure. Body weight was measured using an electronic scale which accommodated wheelchairs.

Each subject was evaluated prior to training using a continuous, progressive intensity $\mathrm{KE}$ exercise protocol to determine a "maximum" KE resistance load with which to begin training. For this test, KE exercise was performed for five repetitions at each of several loads, starting with zero load and progressing in $0.5-\mathrm{kg}$ increments to a maximum of $15.0 \mathrm{~kg}$. The quadriceps muscles were considered fatigued when the KE failed to reach 50 percent of the 70 degree target angle. Since the disuse osteoporosis which accompanies SCI may markedly decrease bone strength, the maximal load was limited to $15.0 \mathrm{~kg}(8,11)$. After the training regimen, this test was repeated (post-test) to determine changes in muscle load resistance capability.

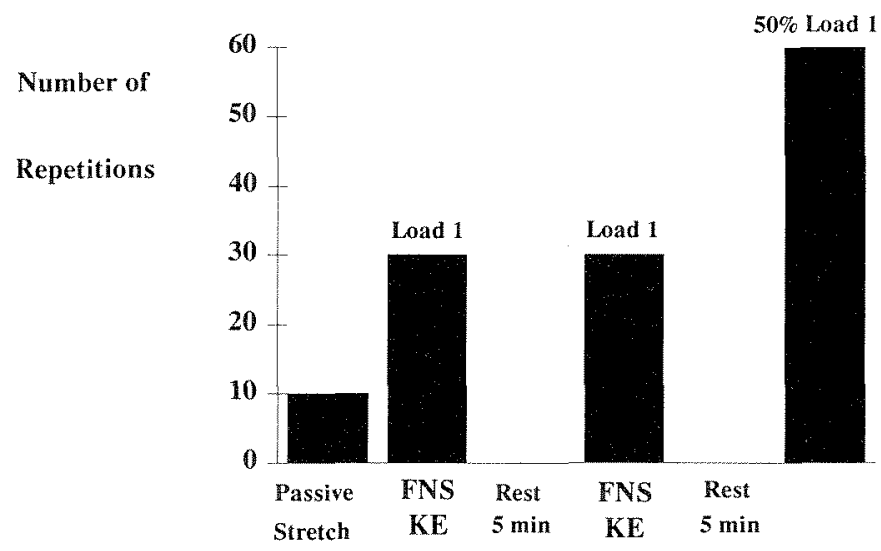

Figure 1.

Diagram showing the progression of activities for FNS-KE exercise training. The overall time of each training session varied depending upon how rapidly the subject's quadriceps muscles fatigued.

\section{Training protocol}

Each subject trained for a total of 36 sessions (approximately 3 times weekly for 12 weeks) using the progressive intensity protocol which is diagramed in Figure 1. Each leg was passively stretched (passive $\mathrm{KE}$ ) for 10 repetitions prior to all training. For the initial session, a load equal to one-fourth of the maximum level achieved during pre-testing was used for two sets of 30 repetitions of FNS-KE separated by a 5-min rest. After another 5-min rest, the load was reduced by one-half, and the exercise continued for 60 repetitions or to fatigue. When the subject was able to complete three consecutive sessions of this protocol, the load resistance was increased by $0.5 \mathrm{~kg}$ for the subsequent session. This progression was continued to a maximum $15.0 \mathrm{~kg}$ load.

\section{Data analysis}

Quadriceps muscle performance (QMP) for individual training sessions was calculated as the number of KE repetitions times the total load (5). The "total load" (for all three exercise bouts) was calculated as the sum of the lever arm weight, the estimated lower leg weight (1), and any additional externally applied load. This total load does not attempt to account for resistance encountered from friction within the weight arm, or for moment arm changes which occur with changing knee angle. Hamstring tightness or spasticity resistances were minimized by positioning each subject in the exercise chair with a hip angle of approximately 110 degrees. The change in QMP was used as a muscle performance index of strength and endurance improvement (QMP index). 
Pre- and post-training evaluations of maximum load, QMP, thigh skinfold thickness, thigh girth, body weight, bone density and passive knee range of motion were compared using dependent $t$ - tests. Leg (left/right) differences were compared for load, QMP, thigh skinfold, thigh girth, and knee range of motion using independent $t$ - tests. The 5 percent significance level was used in all statistical hypothesis testing.

\section{RESULTS}

Although the intended training regimen was 3 times per week during a 12-week period, the actual total time for training varied from 12 to over 18 weeks ( 82 to 131 days). Average attendance rate was 2.5 sessions per week ( 36 sessions/ 14.2 weeks). Two subjects who completed the training did not complete both pre- and post-training tests, but their load resistance progressions were reported for the 36session training period. QMP for each leg of all subjects increased significantly over the training period (right $\bar{x}=$ 1156.0 versus $1624.8 \mathrm{~kg} \cdot$ reps, left $\overline{\mathrm{x}}=1127.3$ versus 1721.1 $\mathrm{kg} \cdot$ reps). Comparison of right and left legs showed no significant difference in QMP.

The QMP progression over the 36-session training period for the left leg of selected subjects is shown in Figures $\mathbf{2} \mathbf{a}, \mathbf{2 b}$, and $\mathbf{2} \mathbf{c}$. The relative change in QMP (postminus pre-training levels) was generally higher for subjects with more recent injuries, as illustrated in an SCI individual who was 1.6 years postinjury (Figure 2a). However, even those with long-term SCI also improved their QMP, as seen in an SCI individual who was 17 years postinjury (Figure $\mathbf{2 b}$ ). Four subjects exhibited a ceiling effect imposed by the protocol and safety precautions, as illustrated in Figure 2c. These individuals were strong enough to progress at the fastest rate allowed by the pro-

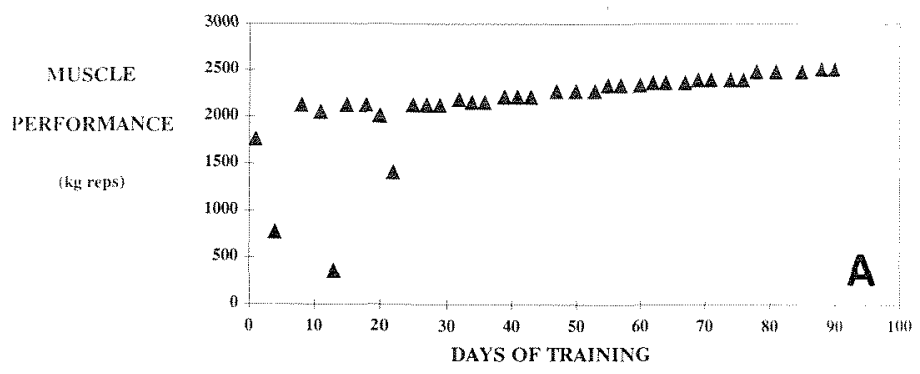

Figure 2A.

Quadriceps muscle performance (QMP) score during FNS-KE exercise showing the progress of a 41-year-old female subject with a recent incomplete $\mathrm{SCl}(1.6 \mathrm{yr}$ post- $\mathrm{SCI})$ at the $\mathrm{C}_{5}$ level.

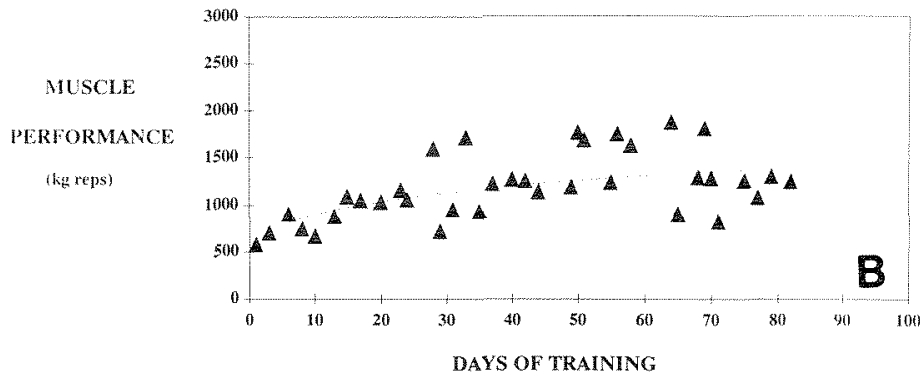

Figure 2B.

Quadriceps muscle performance (QMP) score during FNS-KE exercise showing the progress of a 41-year-old male subject who demonstrated QMP increases despite a long-term incomplete $\mathrm{SCI}$ (15.3 yr post-SCI) at the $\mathrm{C}_{7}$ level.

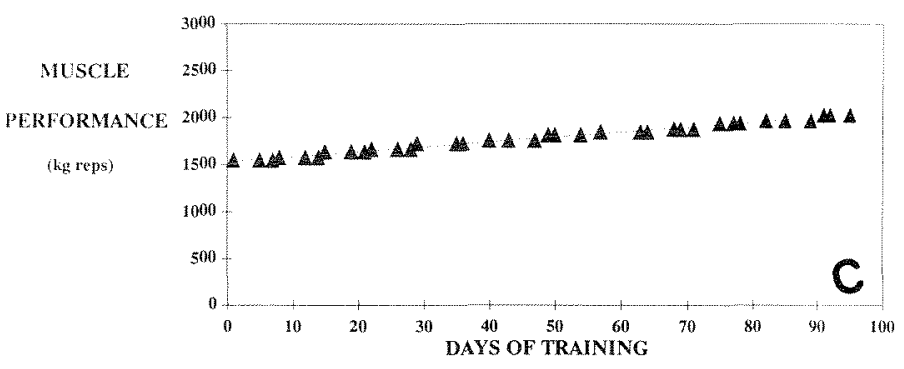

Figure 2C.

Quadriceps muscle performance (QMP) score during FNS-KE exercise showing the progress of a 48 -year-old male subject ( 2.4 yrs postSCI, $\mathrm{T}_{6}$ level, complete $\mathrm{SCI}$ ) who achieved the maximum QMP progression allowed by the training protocol, which was probably less than full capability.

Table 2.

Pre- and post-FNS KE training evaluation data.

\begin{tabular}{lrrrrr}
\hline & N & \multicolumn{2}{c}{ Left Leg } & \multicolumn{2}{c}{ Right Leg } \\
& & Pre & Post & Pre & Post \\
\hline QMP (kg-reps) & 12 & 1127.3 & $1721.1^{*}$ & 1156.0 & $1624.8^{*}$ \\
& & \pm 431.4 & \pm 457.2 & \pm 482.3 & \pm 591.5 \\
& & 5.8 & $11.3^{*}$ & 6.1 & $11.4^{*}$ \\
Maximum load & 10 & & & \\
$\quad$ resistance (kg) & & \pm 2.6 & \pm 3.2 & \pm 2.3 & \pm 3.4 \\
Thigh skinfold & 5 & 24.7 & 17.6 & 23.4 & 17.4 \\
$\quad$ thickness (mm) & & \pm 13.5 & \pm 7.1 & \pm 13.1 & \pm 7.2 \\
Thigh girth & 10 & 48.2 & 46.8 & 48.6 & 46.7 \\
$\quad$ (cm) & & \pm 6.5 & \pm 5.3 & \pm 7.1 & \pm 5.3 \\
Knee ROM & 8 & 132.5 & $143.8^{*}$ & 133.5 & $145.8^{*}$ \\
$\quad$ (deg) & & \pm 17.3 & \pm 13.2 & \pm 16.9 & \pm 11.7 \\
\hline
\end{tabular}

Means and standard deviations are shown.

Asterisk indicates significance at the .05 level. 


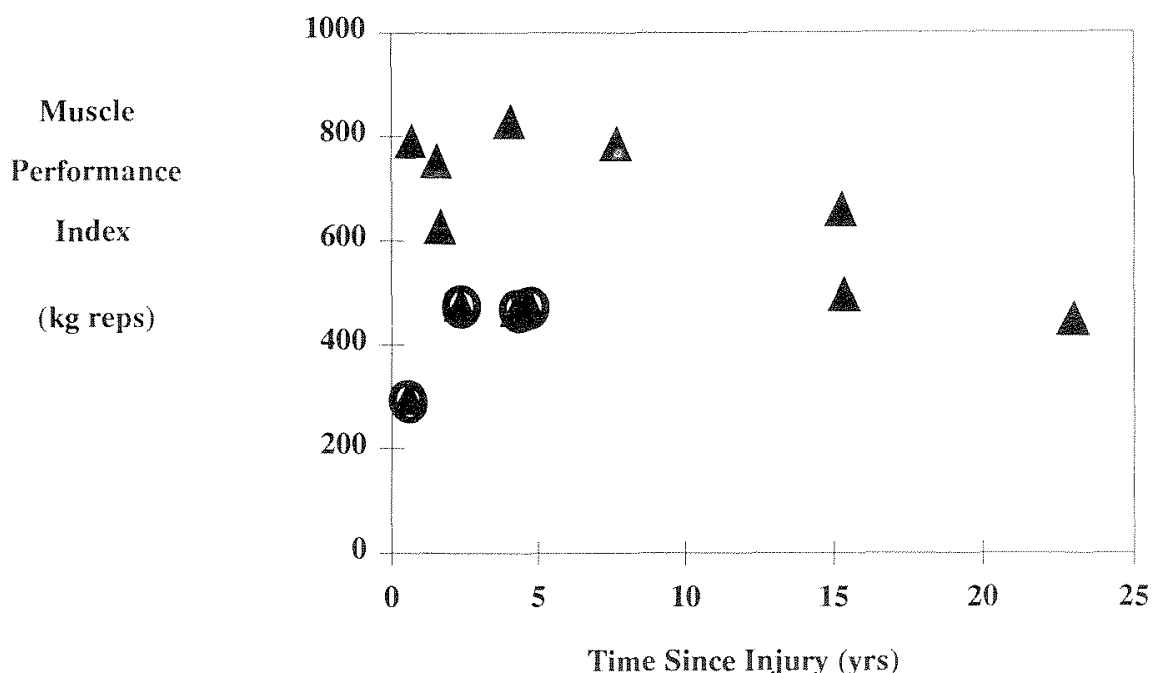

Figure 3.

Quadriceps muscle performance (QMP) index versus time since $\mathrm{SCl}$ for all 12 subjects, with the four subjects who demonstrated a "ceiling effect" circled. tocol. As a result, the recorded change in QMP for these individuals was low relative to the other subjects, and most likely lower than their true capability. Figure 3 shows left QMP index plotted against time-since-injury for all subjects with the four stronger subjects circled. Three of these subjects were incomplete injuries, and all achieved the maximum increase allowed by the protocol. They would probably have continued to increase if the training period had been extended.

Mean body weight decreased following training $(\overline{\mathrm{x}}=$ 67.6 versus $66.9 \mathrm{~kg}$ ), but not significantly. Additional preand post-training evaluation data are shown in Table $\mathbf{2}$. Maximum load resistance and passive knee ROM were significantly higher following training for both legs. Although those subjects who could be measured $(n=5)$ showed a consistent decrease in thigh skinfold thickness after training,

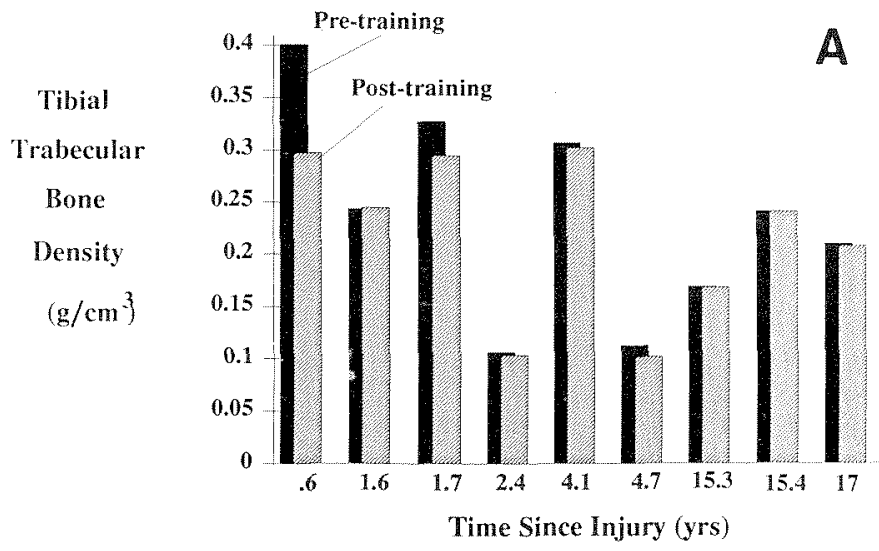

Figure 4A.

Pre- and post-training measurements of tibial trabecular bone (TBD) for nine of the $12 \mathrm{SCI}$ subjects. The measurements are sequenced from shortest to longest time since injury.

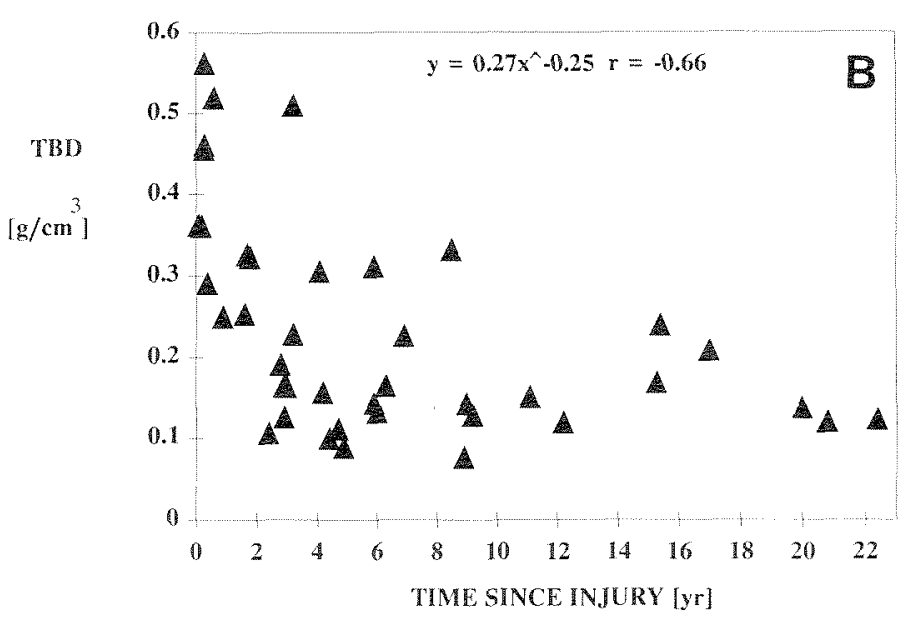

Figure 4B.

Plot showing the tibial trabecular bone density (TBD) versus time since $\mathrm{SCI}$ for a cross-section of 40 subjects before training.

the measurements were not significantly different. Thigh girths decreased, but were not significantly altered by FNS$\mathrm{KE}$ training for either leg.

Pre- and post-training tibial bone density (TBD) measurements were obtained for nine of the subjects (three paraplegics, six quadriplegics). Pre- and post-training TBD measurements for each subject sequenced by time-sinceSCI are shown in Figure 4a. The rate of bone loss (postminus pre-training TBD divided by training time) ranged from 0.0003 to $0.1379 \mathrm{~g} / \mathrm{cm}^{3} / \mathrm{yr}(\overline{\mathrm{x}}=0.0317 \pm 0.0506)$. For comparison purposes, a plot of TBD versus time-sinceinjury for a cross-section of $40 \mathrm{SCI}$ subjects is shown in Figure $4 \mathrm{~b}$. Using the regression equation derived from these data, the "expected" TBD relative to the time-sinceinjury of the individual and the expected rate of bone loss 
Journal of Rehabilitation Research and Development Vol. 28 No. 4 Fall 1991

can be calculated. Seven of the nine subjects had greater TBD than that predicted, and eight subjects experienced a bone loss rate which was lower than predicted. The youngest (19 years old) and most recently injured subject ( 0.8 years post-SCI) was the only one who demonstrated a bone loss rate higher than what was predicted.

\section{DISCUSSION}

The rationale for the training protocol used included the following concepts. Since weights can be added to the lever arms of the KE chair to provide progressive resistance exercise, the principle of "overload" can be followed for quadriceps strengthening (16). The protocol was designed to include both strength and endurance training principles. If the resistance is maintained at a low level and the repetitions are high, the activity promotes endurance more than strength training. The specific protocol used provided effective concentric and eccentric conditioning against gravity and against progressive external load resistance.

Based on the results of this study, the exercise protocols and instrumentation were found to provide several benefits for the SCI individual. Increases in QMP (a measurement which includes both strength and endurance improvements) appear to reflect the effectiveness of the training protocol. Attendance of less than three times per week also did not appear to reduce training effects, since all subjects improved their QMP regardless of their attendance frequency. The testing and training protocols developed for this study appear to be safe and effective, and QMP increased markedly in those SCI subjects whose paralyzed muscles respond to FNS, especially those more recently injured. Findings indicate that the FNS-KE exercise employed in this study provided increased strength in paralyzed muscles with minimal risk for the $\mathrm{SCI}$ individual.

Overall increases in QMP with FNS-KE exercise concurs with previous work by Faghri, et al. (5), who used different training protocols, instrumentation, and a shorter training period (7 weeks). However, the present study incorporated total load for its QMP calculation, and, therefore, demonstrated final QMP levels which were substantially higher. Although not reported by Faghri, et al. (5), a QMP index (change in QMP) derived from those data appears to be substantially lower than in the present study. The protocols and instrumentation of the present study, therefore, appear to provide more effective training for given loads calculated in the same manner.

The increase in levels of absolute external resistance with training concurs with other studies using different instrumentation and training protocols, although the levels achieved in the present study were much higher. Average final external resistance in the present study was $11 \mathrm{~kg}$, compared to $4 \mathrm{~kg}$ in the study by Ragnarsson, et al. (21), $5.5 \mathrm{~kg}$ in the study by Gruner, et al. (11), $6 \mathrm{~kg}$ in the study by Faghri, et al. (5), and $9 \mathrm{~kg}$ in the study by Fournier, et al. (7). This difference supports the use of a higher ceiling for external load which is still within safe limits. Of particular safety concern is the potential injury which may occur due to the strengthening of paralyzed muscles beyond the structural integrity of osteoporotic bone, and the possibility of altered biomechanics of the motion which results from stimulated muscle contractions. The higher maximum resistance load $(15 \mathrm{~kg})$, used for both evaluation and training in this study, appeared to be well within safe limits for those SCI subjects performing FNS-KE exercise in this study. However, SCI individuals with severe osteoporosis may require a lower maximum load resistance to prevent injury.

Based on individual QMP graphs (examples are shown in Figures $\mathbf{2 a}, \mathbf{2 b}$, and $\mathbf{2 c}$ ), transitory decreases in performance appeared to coincide with initial increases in external load resistance. The training protocol required the resistive load to be increased after successfully completing the required number of repetitions for three consecutive training sessions. However, for many subjects, the 0.5 $\mathrm{kg}$ increment in resistive load resulted in fewer completed repetitions and a net diminished QMP score. Interestingly, the subjects did not appear to reach a plateau during the training period. Longer FNS-KE training periods may elicit increased QMP, although not necessarily at the same rate. Further investigation is needed regarding the upper limit for performance increases, the rate of detraining, and the effects of medication on these results.

Another significant finding of this study was increased passive knee ROM, which may aid in the prevention of joint contractures and allow easier performance of activities of daily living. The lack of significant change in thigh skinfold thickness may be related to the small number of subjects $(n=5)$ who could be measured. Those subjects who could not be measured exhibited a condition where the thigh skin could not be "pinched," possibly due to increased fluid content (edema) or soft tissue changes. The frequency of this problem indicates the need for alternative methods of measuring body composition in SCI populations (e.g., bioimpedance methods).

Previous studies have demonstrated increased thigh girths, and suggested that FNS-KE exercise may increase quadriceps size $(5,7,11,21)$. The lack of significant change in thigh girth found in the present study may reflect changes 
Table 3.

SCI lesion level and completeness, knee reflex tone (on a scale of 0 to 4), antispasticity medication, and quadriceps muscle performance index (post- minus pre-training QMP). Subjects are ordered by their QMP index (highest to lowest).

\begin{tabular}{lclc}
\hline \multicolumn{1}{c}{ SCI Lesion } & Tone & \multicolumn{1}{c}{ Medication } & QMP Index \\
\hline $\mathrm{T}_{1}$ complete & 3.0 & Ditropan & 829 \\
$\mathrm{~T}_{10}$ incomplete & 2.0 & Dantrium & 792 \\
$\mathrm{C}_{7}$ complete & 2.5 & none & 786 \\
$\mathrm{C}_{5}$ incomplete & 4.0 & Valium, Lioresal & 754 \\
$\mathrm{C}_{7}$ incomplete & 4.0 & Valium & 660 \\
$\mathrm{C}_{6}$ incomplete & 4.5 & Valium, Lioresal & 628 \\
$\mathrm{C}_{6}$ complete & 4.0 & none & 498 \\
$\mathrm{~T}_{6}$ complete & 3.0 & Valium, Ditropan & 480 \\
$\mathrm{C}_{4}$ incomplete & 3.0 & Valium, Lioresal & 479 \\
$\mathrm{C}_{5}$ complete & 3.0 & none & 470 \\
$\mathrm{~T}_{5}$ incomplete & 3.0 & none & 450 \\
$\mathrm{C}_{5}$ incomplete & 4.5 & none & 300 \\
\hline \hline
\end{tabular}

in body composition, as opposed to a lack of quadriceps hypertrophy, such as decreased subcutaneous fat or fluid content (edema) of the thigh. In addition, body weight decreased, although the amount was not significant. The combination of decreasing body weight, body fat, and/or edema may have obscured an increase in quadriceps size. The significant increase in maximum load resistance strongly suggests that quadriceps strength was increased despite the lack of girth change.

Because contraction of muscles contributes to the mechanical stresses necessary to maintain bone strength and stiffness (via the tendon-bone attachments), electricallyinduced muscle contraction may affect bone density $(14,17)$. Within the constraints of the sample size $(n=9)$ included for bone density measurements, the TBD findings suggest that this form of exercise may be beneficial to the bone located in close proximity to the exercising muscles. For the seven subjects whose bone density decreased during the training period, the rate of bone loss was substantially less than would be expected based on our earlier crosssectional study of $40 \mathrm{SCI}$ subjects (23). In this previous study, a bone loss rate of $0.15 \mathrm{~g} / \mathrm{cm}^{3} / \mathrm{yr}$ was observed in the first 2 years after injury in subjects who were not involved in FNS exercise. The highest bone loss rate in the present study $\left(0.138 \mathrm{~g} / \mathrm{cm}^{3} / \mathrm{yr}\right)$ was found in a subject who was 0.8 years post-SCI. However, for two subjects with
SCI of 1.6 and 15.4 years' duration, no additional bone loss occurred during the training period. Based on our earlier work, even those with long-term SCI would be expected to demonstrate some bone loss. Within the limitations of this study, these findings suggest that FNS-KE exercise may provide a deterrent to tibial bone loss in SCI individuals.

Other factors which can potentially influence individual subject performance with training include previous training status and medications. Only one subject $\left(\mathrm{C}_{6}\right.$ complete $\mathrm{SCI}$ male) had previous FES exercise training prior to participation in this study. For one year prior to this study, the subject had regularly trained on a leg cycle ergometer. However, his initial load level and progress in the knee extension exercise of the present study was very similar to that of another untrained male subject with similar physical involvement $\left(\mathrm{C}_{5}\right.$ complete $\left.\mathrm{SCI}\right)$. The initial and final external loads for the trained subject were 3.5 and $9.5 \mathrm{~kg}$ compared to 2.5 and $7 \mathrm{~kg}$ for the untrained subject, respectively. Therefore, previous FES training appeared to have little effect on quadriceps muscle training in this case.

Seven of the 12 subjects who participated in the study were taking antispasticity medications during training. Table 3 shows-for each subject-the change in left leg QMP (post- minus pre-training levels), the responses to lower extremity reflex testing (from the neurological exam), and the antispasticity medication taken. The patellar tendon reflex response is graded from zero to $4+$, with zero being considered hypotonic, 2 normal, and $4+$ hypertonic (clonus). The mean change in QMP was actually higher for the group taking medication (660 versus $501 \mathrm{~kg} \cdot \mathrm{reps}$ ), but not significantly. The medications may have aided muscle strength and endurance training by allowing more isolated and controlled muscle activity and by decreasing resistance from antagonistic hamstring muscle groups.

\section{CONCLUSIONS}

FNS-induced KE exercise provides a number of musculoskeletal benefits for the SCI individual whose muscles respond to electrical stimulation. These include increased quadriceps muscle strength/endurance, and passive knee range of motion. In addition, FNS-KE exercise appeared to decrease the rate of tibial bone loss in our SCI subjects. From our results, this exercise can potentially be most helpful for recently injured SCI individuals in order to maintain muscle integrity and joint ROM and to retard tibial bone loss. These benefits are especially important to those SCI individuals who may experience 
some return of voluntary function or who are preparing for more strenuous FNS activities such as leg cycling, standing, or ambulation.

\section{ACKNOWLEDGMENT}

Sincere appreciation is expressed to Randy Shively for technical assistance during data collection.

\section{REFERENCES}

1. Clauser CE, McConville JT, Young JW: Weight, volume, and center of mass of segments of the human body. In AMRL-TR-69-70: Aerospace Medical Research Laboratories, Dayton, OH: WrightPatterson Air Force Base, 1969.

2. Collins SR, Glaser RM: Comparison of aerobic metabolism and cardiopulmonary responses for electrically induced and voluntary exercise. In Proceedings of the 8th Annual RESNA Conference, Memphis, TN, 391-393, 1985.

3. Ezenwa BN, Glaser RM, Couch W, Figoni SF, Rodgers MM: Adaptive control of functional neuromuscular stimulation-induced knee extension exercise. J Rehabil Res Dev 28(4):1-8, 1991.

4. Ezenwa BN, Glaser RM, Couch W, Figoni SF, Rodgers MM, Suryaprasad AG, Mathews T: Automated adaptive equalization system for asynchronous FNS-induced knee extension exercise for SCI subjects. In Proceedings of the 13th Annual RESNA Conference, Washington, DC, 159-160, 1990.

5. Faghri PD, Glaser RM, Figoni SF, Miles DS, Gupta SC: Feasibility of using two FNS exercise modes for spinal cord injured patients. Clin Kinesiol 43:62-68, 1989.

6. Figoni SF, Glaser RM, Rodgers MM, Hooker SP, Ezenwa BN, Collins SR, Mathews T, Suryaprasad AG, Gupta SC: Acute physiologic responses of spinal cord injured individuals to functional neuromuscular stimulation-induced knee extension exercise. J Rehabil Res Dev 28(4):9-18, 1991.

7. Fournier A, Goldberg M, Green B, Brucker B, Petrofsky J, Eismont $\mathbf{F}$, Quencer $\mathbf{R}$, Sosenko J, Pina I, Shebert R, Kessler KK, MacDonald A, Fiore P, Burnett B: A medical evaluation of the effects of computer induced muscle stimulation in paraplegic patients. Orthopaedics 7:1129-1133, 1984.

8. Glaser RM: Physiologic aspects of spinal cord injury and functional neuromuscular stimulation. Cent Nerv Syst Trauma $3: 49-62,1986$

9. Glaser RM, Collins SR, Horgan HR: An electrical stimulator for exercising paralyzed muscles. In Proceedings of the 10th Annual RESNA Conference, San Jose, CA, 597-599, 1987.

10. Glaser RM, Collins SR, Strayer JR, Glaser M: A closed-loop stimulator for exercising paralyzed muscles. In Proceedings of the 8th Annual RESNA Conference, Memphis, TN, 388-390, 1985.

11. Gruner JA, Glaser RM, Feinberg SD, Collins SR, Nussbaum NS: A system for evaluation and exercise-conditioning of paralyzed leg muscles. J Rehabil Res Dev 20(1):21-30, 1983.

12. Hangartner TN, Gabel SJ, Rodgers MM, Barre PS, Glaser RM: Ultra precise computed tomography scanner for measurement of bone density in extremities. In Proceedings of the 35th Annual Orthopaedic Research Society Meeting, 104, 1989.
13. Kralj A, Bajd T, Turk R: Electrical stimulation providing functional use of paraplegic patient muscles. Med Prog Tech 7:3-9, 1980.

14. Lew RD: The effects of FNS on disuse osteoporosis. In Proceedings of the 10th Annual RESNA Conference, San Jose, CA, 616-617, 1987

15. Long C: Congenital and traumatic lesions of the spinal cord. In Handbook of Physical Medicine and Rehabilitation, 2nd ed., 566-578, F.H. Krusen (Ed.). Philadelphia: W.B. Saunders Company, 1971.

16. McArdle WD, Katch FI, Katch VL: Exercise Physiology, 2nd ed., 266-270. Philadelphia: Lea \& Febiger, 1981.

17. Nordin M, Frankel VH: Biomechanics of whole bones and bone tissue. In Basic Biomechanics of the Skeletal System, 15-60. Philadelphia: Lea \& Febiger, 1980.

18. Peckham PH, Mortimer JT, Marsolais EB: Alteration in the force and fatigability of skeletal muscle in quadriplegic humans following exercise induced by chronic electrical stimulation. Clin Orthop Rel Res 114:326-334, 1976.

19. Petrofsky JS, Heaton HH, Phillips CA: Leg exerciser for training of paralyzed muscle by closed-loop control. Med Biol Eng Comput 298-303, 1984.

20. Petrofsky JS, Phillips CA: Active physical therapy: A modern approach to rehabilitation therapy. I Neurol Orthop Med Surg 4:165-173, 1983.

21. Ragnarsson KT, Pollack S, O'Daniel W, Edgar R, Petrofsky JS, Nash MS: Clinical evaluation of computerized functional electrical stimulation after spinal cord injury: A multicenter pilot study. Arch Phys Med Rehabil 69:672-677, 1988.

22. Robinson CJ, Bolam JM, Kett NA, Engelmeier NA, Fruin RC, Nemchausky BA: Predictive factors for quadriceps reconditioning via electrical stimulation. In Proceedings of the 9th Congress on External Control of Human Extremities, Dubrovnik, Yugoslavia, 111-116, 1987.

23. Rodgers MM, Hangartner TN, Barre PS, Glaser RM, Gabel SJ: Tibial trabecular bone density versus time since spinal cord injury. In Proceedings of the 12th Annual RESNA Conference, New Orleans, LA, 403-404, 1989.

\section{APPENDIX}

\section{Explanatory abbreviations}

FNS functional neuromuscular stimulation

KE knee extension

QMP quadriceps muscle performance

ROM range of motion

$\mathrm{SCI}$ spinal cord injury, spinal cord injured

SD standard deviation

TBD trabecular bone density

$\overline{\mathrm{X}}$ mean 